\title{
Preparation and Crystal Structure of Two Bicyclo[1.1.0]butan-2-ones. A Hybrid Oxyallyl-Cyclopropanone Motif
}

\author{
Surbhi Bhargava, Jianjun Hou, Masood Parvez, and Ted Sorensen \\ Department of Chemistry, University of Calgary, 2500 University Drive, Calgary, \\ Alberta T2N1N4 Canada
}

Supporting Information

\section{Experimental Preparation Details and NMR Data for 4 and 5}
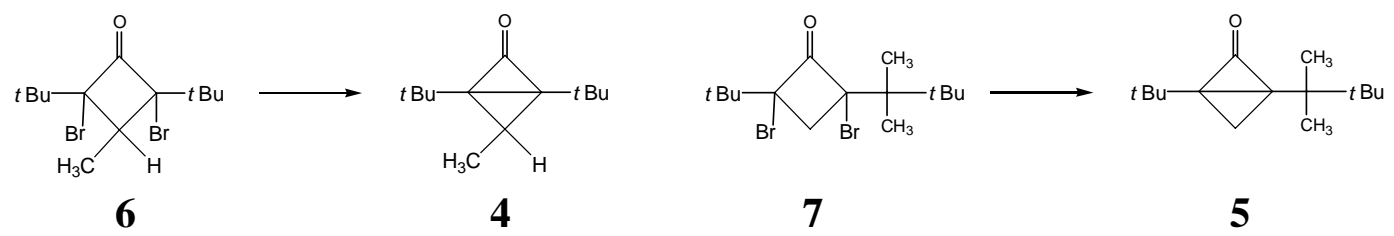

Preparation and Recrystallization of 1,3-di-t-Bu-4-methylbicyclo[1.1.0]butan-2-one 4

Ketone $6(100 \mathrm{mg}, 0.282 \mathrm{mmol})$ in $1 \mathrm{~mL}$ of dry dichloromethane was added dropwise by syringe into a stirred solution of $\mathrm{PPN}^{+} \mathrm{Cr}(\mathrm{CO})_{4} \mathrm{NO}^{-\S}(209 \mathrm{mg}, 0.282 \mathrm{mmol})$ in $1 \mathrm{~mL}$ of dry dichloromethane which was contained in a small Schlenk tube protected with a septum and under $\mathrm{N}_{2}$, in a $-78^{\circ} \mathrm{C}$ acetone-dry ice bath. After stirring for $20 \mathrm{~min}, 15 \mathrm{~mL}$ of dry pentane was slowly added by syringe. The mixture was stirred for a further $10 \mathrm{~min}$ and then let stand for $30 \mathrm{~min}$. The supernatant was transferred by a double ended needle to a clean Schlenk tube also equipped with a septum under $\mathrm{N}_{2}$, in a $-78^{\circ} \mathrm{C}$ acetone-dry ice bath. The solvent was evaporated at $c a .-40$ to $-50^{\circ} \mathrm{C}$ under vacuum $(c a .0 .01 \mathrm{~mm} \mathrm{Hg}$ ) to leave a waxy solid. The residue was redissolved in $5 \mathrm{~mL}$ of pentane $\left(\right.$ at $c a .-50^{\circ} \mathrm{C}$ ), and the solution let stand for $10 \mathrm{~min}$. The solution was filtered by quickly drawing the cold solution into a cold syringe, replacing the needle with a syringe filter, and then reintroducing the filtered solution into a clean cold Schlenk tube. The solvent was evaporated as above to leave a solid residue. $1 \mathrm{~mL}$ of THF was added to dissolve the residue and vacuum was applied again at $c a .-50^{\circ} \mathrm{C}$. Small crystals of $\mathbf{4}$ could be obtained with the slow evaporation of pentane. The crystals could be kept under $\mathrm{N}_{2}$ in dry ice for several days. A watch glass was cooled by blowing liquid nitrogen across it for a short time and then several pieces of dry ice were put on it to keep it cold. Crystals from the cold Schlenk tube were placed on the watch glass, and immediately slurried with precooled Paratone 8277 oil (Exxon). Microscopic examination allowed a suitable crystal to be found and mounted on a Nonius Kappa CCD diffractometer, using Mo-K $\alpha$ radiation.. The data were collected at about $173 \mathrm{~K}$.

${ }^{1} \mathrm{HNMR}\left(\mathrm{CD}_{2} \mathrm{Cl}_{2,}-38^{\circ} \mathrm{C}\right) 1.12(\mathrm{~s}, 18 \mathrm{H}), 1.30(\mathrm{~d}, \mathrm{~J}=5.3,3 \mathrm{H}), 2.90(\mathrm{q}, \mathrm{J}=5.3,1 \mathrm{H})$. ${ }^{13} \mathrm{CNMR}\left(\mathrm{CD}_{2} \mathrm{Cl}_{2},-38^{\circ} \mathrm{C}\right) 23.65\left(\mathrm{CH}_{3}\right), 27.56(t-\mathrm{Bu}), 30.52(\mathrm{C}), 34.45(\mathrm{CH}), 88.25(\mathrm{C})$, $182.08(\mathrm{C}=\mathrm{O})$.

$\S$ Bis(triphenylphosphoranylideneammonium Chromiumtetracarbonynitrosyl ${ }^{1}$ 


\section{Preparation and Recrystallization of 1-t-Bu-3-(1,1,2,2-}

tetramethylpropyl)bicyclo[1.1.0]butan-2-one 5

Ketone 7 (100mg, $0.262 \mathrm{mmol}$ ) in $1 \mathrm{~mL}$ of dry dichloromethane was added dropwise by syringe into a stirred solution of $\mathrm{PPN}^{+} \mathrm{Cr}(\mathrm{CO})_{4} \mathrm{NO}^{-}(194 \mathrm{mg}, 0.262 \mathrm{mmol})$ in $1 \mathrm{~mL}$ of dry dichloromethane which was contained in a small Schlenk tube protected with a septum and under $\mathrm{N}_{2}$ in a $-78^{\circ} \mathrm{C}$ acetone-dry ice bath. The following steps were similar to those reported for $\mathbf{4}$ except the solvent used for crystallization was THF and the X-ray data were collected at about $153 \mathrm{~K}$.

${ }^{1} \mathrm{H}-\mathrm{NMR}\left(\mathrm{CD}_{2} \mathrm{Cl}_{2},-38^{\circ} \mathrm{C}\right) 0.81(\mathrm{~s}, 3 \mathrm{H}), 0.94(\mathrm{~s}, 9 \mathrm{H}), 1.09(\mathrm{~s}, 3 \mathrm{H}), 1.12(\mathrm{~s}, 9 \mathrm{H}), 2.45(\mathrm{~s}$, $1 \mathrm{H}), 2.47(\mathrm{~s}, 1 \mathrm{H}){ }^{13} \mathrm{C}$ NMR $\left(\mathrm{CD}_{2} \mathrm{Cl}_{2},-38^{\circ} \mathrm{C}\right) 20.62\left(\mathrm{CH}_{3}\right), 21.08\left(\mathrm{CH}_{3}\right), 25.73(\mathrm{br}, t-\mathrm{Bu})$, $27.66(t-\mathrm{Bu}), 27.99\left(\mathrm{CH}_{2}\right), 30.26(\mathrm{C}) 37.53(\mathrm{C}), 38.84(\mathrm{C}), 79.51(\mathrm{C}), 83.77(\mathrm{C}), 180.89$ $(\mathrm{C}=\mathrm{O})$. Impurity peaks in the ${ }^{13} \mathrm{C}$ NMR at $\delta 124-133$ are from the $\mathrm{PPN}^{+}$cation. Small hydrocarbon peaks from the work-up are difficult to completely remove at the low temperatures involved.

Preparation of $c i s-2,4-D i b r o m o-2,4-d i-t-b u-3-m e t h y l c y c l o b u t a n-1-o n e ~ 6$

A $25 \mathrm{~mL}$ three necked round bottom flask equipped with a condenser, and a gas inlet tube was in turn connected to a safety flask and a $\mathrm{HBr}$ generator. 2,4-di-t-Bu-3methylcyclobutanone 12 (100mg, 0.51mmol), carbon tetrachloride (15mL) and $\mathrm{Br}_{2}$ (260mg; 1.63mmol) was added. Into this a slow stream of $\mathrm{HBr}$ was bubbled continuously. After $14 \mathrm{~h}$, the monobrominated compound was the main product as monitored by NMR. The monobrominated product solution was transferred to a thick walled glass reaction vial $(50 \mathrm{~mL}), \mathrm{Br}_{2}(130 \mathrm{mg}, 0.82 \mathrm{mmol})$ was added, the reaction vial was flame-sealed after $\mathrm{HBr}$ was bubbled into the reaction mixture. The vial was placed in an oven preset to $100^{\circ} \mathrm{C}$ for 4 days. The solvent was removed from the reaction mixture and residue was purified by silica gel column chromatography with the eluting solvent dichloromethane: hexane $=1: 9$, giving $110 \mathrm{mg}(0.31 \mathrm{mmol})$ of $6(61 \%), \mathrm{mp}$. dec. $>86^{\circ} \mathrm{C}$; ${ }^{1} \mathrm{H}-\mathrm{NMR}\left(\mathrm{CDCl}_{3}\right) 1.16(\mathrm{~s}, 18 \mathrm{H}), 1.45(\mathrm{~d}, \mathrm{~J}=6.4,3 \mathrm{H}), 2.65(\mathrm{q}, \mathrm{J}=6.4,1 \mathrm{H}) ;{ }^{13} \mathrm{C}-\mathrm{NMR}$ $\left(\mathrm{CDCl}_{3}\right) 19.51\left(\mathrm{CH}_{3}\right), 27.41(t-B u), 37.51(\mathrm{CH}), 38.61(\mathrm{C}), 85.25(\mathrm{C}-\mathrm{Br}), 202.39(\mathrm{C}=\mathrm{O})$; high resolution mass for $6, \mathrm{C}_{13} \mathrm{H}_{22} \mathrm{O}^{79} \mathrm{Br}^{81} \mathrm{Br}$, found 353.99904, calcd 354.00169. The $\mathrm{CH}_{3}$ group at $\mathrm{C} 3$ is assigned as cis to the $\mathrm{Br}$ groups on steric grounds.

Preparation of trans-2,4-Dibromo-2-t-bu-4-(1,1,2,2-tetramethylpropyl)cyclobutan-1-one 7

A similar procedure to that used to prepare 6 was employed. 2-t-Bu-4-(1,1,2,2tetramethylpropyl)cyclobutan-1-one 9 (720 $\mathrm{mg} ; 3.21 \mathrm{mmol})$, carbon tetrachloride $(40 \mathrm{ml})$ and $\mathrm{Br}_{2}(1.54 \mathrm{~g} ; 9.63 \mathrm{mmol})$ were used. After $13 \mathrm{~h}$, the monobrominated compound was the main product as monitored by NMR. The monobrominated product was converted to the dibromide using the same procedure as for 6 . The eluting solvent for 7 was dichloromethane:pentane $=1: 15$, giving $724 \mathrm{mg}(1.90 \mathrm{mmol})$ of $6(59 \%), \mathrm{mp} 78-80{ }^{\circ} \mathrm{C} ;{ }^{1} \mathrm{H}$ $\operatorname{NMR}\left(\mathrm{CDCl}_{3}\right) 1.10(\mathrm{~s}, 9 \mathrm{H}), 1.26(\mathrm{~s}, 9 \mathrm{H}), 1.30(\mathrm{~s}, 3 \mathrm{H}), 1.33(\mathrm{~s}, 3 \mathrm{H}), 3.08(\mathrm{~d}, \mathrm{~J}=16.0,1 \mathrm{H})$, $3.36(\mathrm{~d}, \mathrm{~J}=16.0,1 \mathrm{H}) ;{ }^{13} \mathrm{C} \mathrm{NMR}\left(\mathrm{CDCl}_{3}\right) 22.91\left(\mathrm{CH}_{3}\right), 24.17\left(\mathrm{CH}_{3}\right), 27.69(t-\mathrm{Bu}), 28.50$ $\left(t\right.$-Bu), $36.83(\mathrm{C}), 38.60(\mathrm{C}) 43.26(\mathrm{C}), 47.00\left(\mathrm{CH}_{2}\right), 82.76(\mathrm{C}-\mathrm{Br}), 84.15(\mathrm{C}-\mathrm{Br}), 197.72$ 
(CO); high resolution mass for $\mathrm{C}_{11} \mathrm{H}_{18} \mathrm{O}^{79} \mathrm{Br}\left(\mathrm{M}^{+\cdot}-\mathrm{C}_{4} \mathrm{H}_{8}-\mathrm{Br}\right)$, found 245.05168, calcd 245.05410; for $\mathrm{C}_{11} \mathrm{H}_{18} \mathrm{O}^{81} \mathrm{Br}$, found 247.05415, calcd 247.05206.

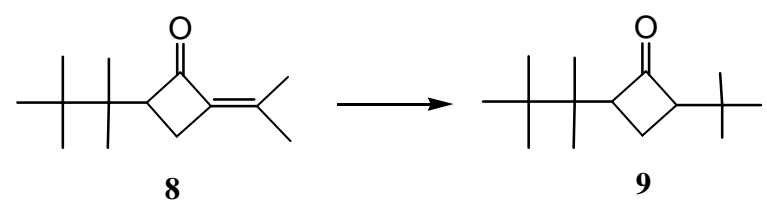

Preparation of 2-(1-Methylethylidene)-4-(1,1,2,2-tetramethylpropyl)cyclobutan-1-one 8

t-Butylmagnesium chloride was prepared in ether solvent $(50 \mathrm{~mL})$ from $5.55 \mathrm{~g},(0.060$ mol) of the chloride. After completion, freshly distilled THF $(60 \mathrm{~mL})$ was added and the mixture cooled to $0^{\circ} \mathrm{C}$. Copper(I) iodide, ca. $0.1 \mathrm{~g}$, was added and stirring continued for $2 \mathrm{~h}$. Then, di-2,4-(1-methylethylidene)cyclobutanone, ${ }^{2} 3.0 \mathrm{~g}(0.020 \mathrm{~mol})$, in THF was added over a period of $1 \mathrm{~h}$ with stirringand then stirred overnight at $20^{\circ} \mathrm{C}$. Work-up with aq $\mathrm{NH}_{4} \mathrm{OH} / \mathrm{NH}_{4} \mathrm{Cl}(\mathrm{pH} 8)$ and ether extraction gave a crude product mixture of 1,4 and 1,2-adduct. Purification by column chromatography, $\mathrm{CH}_{2} \mathrm{Cl}_{2}$ :pentane, 1:3, gave $2.13 \mathrm{~g}$ $(0.010 \mathrm{~mol}),(50 \%)$ of 8 as a colorless liquid. ${ }^{1} \mathrm{H}$ NMR $\left.\left(\mathrm{CDCl}_{3}\right) 0.79(\mathrm{~s}, 9 \mathrm{H}),\right), 0.80$ $(\mathrm{s}, 9 \mathrm{H}), 1.01(\mathrm{~s}, 3 \mathrm{H}), 1.66(\mathrm{~s}, 3 \mathrm{H}), 1.97,(\mathrm{~s}, 3 \mathrm{H}), 2.44-2.48,(\mathrm{~m}, 2 \mathrm{H}), 3.03,(\mathrm{~d}, \mathrm{~d}, 1 \mathrm{H}),{ }^{13} \mathrm{C}$ NMR $18.72\left(\mathrm{CH}_{3}\right), 20.65\left(\mathrm{CH}_{3}\right), 21.31\left(\mathrm{CH}_{3}\right), 21.48\left(\mathrm{CH}_{3}\right), 25.98(\mathrm{tBu}), 26.11,\left(\mathrm{CH}_{2}\right)$, 36.15, (C), 39.91 (C), 62.23, (CH), 140.04 (C), 140.17 (C), 202.17, (CO). high resolution mass for $\mathrm{C}_{14} \mathrm{H}_{24} \mathrm{O}$; found 208.18245, calcd. 208.18272. MS (m/z) $208\left(\mathrm{M}^{+*}, 2\right), 151$ (100), 137 (14), 123 (12), 81 (20), 67 (15), 41 (23).

Preparation of cis-2-tBu-4-(1,1,2,2-tetramethylpropyl)cyclobutanone 9

Ketone 8 (3.6g,0.017mol) was reacted with $\mathrm{CH}_{3} \mathrm{MgI} / \mathrm{CuI}$ under essentially the same conditions used to prepare $\mathbf{8}$ itself. The crude work-up product was a mixture of the 1,2 and 1,4-adduct (GC-MS), which could be separated by column chromatography (1:1 $\mathrm{CH}_{2} \mathrm{Cl}_{2}$ :pentane) to give $2.32 \mathrm{~g}, 0.010 \mathrm{~mol}(61 \%)$ of 9, a clear liquid. ${ }^{1} \mathrm{H} \mathrm{NMR}\left(\mathrm{CDCl}_{3}\right)$ $0.81(\mathrm{~s}, 3 \mathrm{H}), 0.83(\mathrm{~s} .9 \mathrm{H}), 0.91(\mathrm{~s}, 9 \mathrm{H}), 1.01(\mathrm{~s}, 3 \mathrm{H}), 1.79(\mathrm{~m}, 1 \mathrm{H}), 2.02(\mathrm{~m}, 1 \mathrm{H}), 2.82$ $(\mathrm{m}, 1 \mathrm{H}), 3.13(\mathrm{~m}, 1 \mathrm{H}),{ }^{13} \mathrm{C}$ NMR $18.82\left(\mathrm{CH}_{2}\right), 19.13\left(\mathrm{CH}_{3}\right), 21.35\left(\mathrm{CH}_{3}\right), 26.05(\mathrm{tBu})$, $27.41(\mathrm{tBu}), 31.88(\mathrm{C}), 35.84(\mathrm{C}), 39.89(\mathrm{C}), 62.57(\mathrm{CH}), 66.26(\mathrm{CH}), 211.91(\mathrm{CO})$; high resolution mass for $\mathrm{C}_{15} \mathrm{H}_{29} \mathrm{O}$, found 224.21287, calcd 224.21402. MS (m/z) $224\left(\mathrm{M}^{+*}, 1\right)$, 209 (2), 167 (30), $111(20), 83$ (100), 69 (70), 55 (40), 41 (28). The cis stereochemistry was assigned based on that found for the 2,4-di-tBu analog. ${ }^{2}$

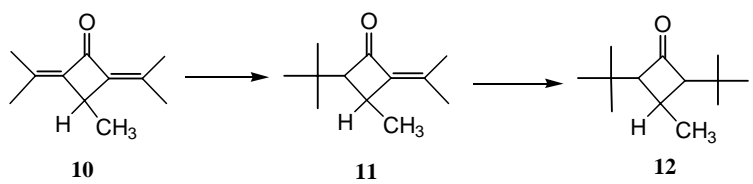

Preparation of 3-Methyl-2,4-di-(1-methylethylidene)cyclobutanone 10

Starting with 3-methylcyclobutanone ${ }^{3}$ the title compound was prepared as a low melting crystalline solid ( $76 \%$ yield after chromatographic separation, $\mathrm{CH}_{2} \mathrm{Cl}_{2}$ ) using a similar procedure to that reported for the cyclobutanone adduct. ${ }^{2}{ }^{1} \mathrm{H} \mathrm{NMR}\left(\mathrm{CDCl}_{3}\right) 1.32$ $(\mathrm{d}, \mathrm{J}=6.7,3 \mathrm{H}), 1.78(\mathrm{~s}, 6 \mathrm{H}), 2.10(\mathrm{~s}, 6 \mathrm{H}), 3.37$ (br q, J=6.7,1H). ${ }^{13} \mathrm{C}$ NMR $18.06\left(\mathrm{CH}_{3}\right)$, 
20.9-21.0 ( $\mathrm{CH}_{3}$ overlapped), $37.10(\mathrm{CH}), 141.19(\mathrm{C}), 145.68(\mathrm{C}), 190.38(\mathrm{CO})$; high resolution mass for $\mathrm{C}_{11} \mathrm{H}_{16} \mathrm{O}$, found 164.11907, calcd 164.12012. MS $(\mathrm{m} / \mathrm{z}) 164\left(\mathrm{M}^{+*}\right.$, 21), 120 (85), 105 (80), 78 (50), 67 (79), 55 (75), 39 (100).

Preparation of trans-2-tBu-3-methyl-4-(1-methylethylidene)cyclobutanone 11

The title compound was prepared from $\mathbf{1 0}$ using the same procedure ${ }^{2}$ as that used to prepare the corresponding cyclobutanone species. A small amount of the 1,2-adduct was removed by column chromatography $\left(\mathrm{CH}_{2} \mathrm{Cl}_{2}\right.$ :hexane) to give the title compound as a colorless liquid, $(1.08 \mathrm{~g}, 6.11 \mathrm{mmol}, 98 \%)$. The trans stereochemistry was assigned on steric grounds. ${ }^{1} \mathrm{H}$ NMR. $\left(\mathrm{CDCl}_{3}\right) 0.96(\mathrm{~s}, 9 \mathrm{H}), 1.32(\mathrm{~d}, \mathrm{~J}=6.8,3 \mathrm{H}), 1.77(\mathrm{~d}, \mathrm{~J}=1.1,3 \mathrm{H})$, $2.06(\mathrm{~d}, \mathrm{~J}=1.8,3 \mathrm{H}), 2.32(\mathrm{~d}, \mathrm{~J}=5.4,1 \mathrm{H}), 2.77(\mathrm{br}, \mathrm{m}, 1 \mathrm{H}) .{ }^{13} \mathrm{C}$ NMR $20.03\left(\mathrm{CH}_{3}\right), 20.44$ $\left(\mathrm{CH}_{3}\right), 21.31\left(\mathrm{CH}_{3}\right), 27.44(\mathrm{tBu}), 31.63(\mathrm{CH}), 32.77(\mathrm{C}), 74.65(\mathrm{CH}), 142.15(\mathrm{C}), 144.30$ (C), $190.28(\mathrm{CO})$; high resolution mass for $\mathrm{C}_{12} \mathrm{H}_{20} \mathrm{O}$, found 180.15096 , calcd 180.15142 MS (m/z) $180\left(\mathrm{M}^{+*}, 60\right) 165(45), 123(81), 109(46), 82$ (100), 67 (80), 41 (30).

Preparation of 2,4-ditBu-3-methylcyclobutanone 12

Ketone 11 was again reacted with $\mathrm{CH}_{3} \mathrm{MgI} / \mathrm{CuI}$ to give a mixture of 1,2 and 1,4adducts. Separation by column chromatography $\left(\mathrm{CH}_{2} \mathrm{Cl}_{2}\right.$ :hexane,1:2) gave the title ketone, $(0.71 \mathrm{~g}, 3.6 \mathrm{mmol}, 65 \%)$ as a white solid, $\mathrm{mp} 42-44^{\circ} \mathrm{C}$. The ${ }^{1} \mathrm{H}$ and ${ }^{13} \mathrm{C}$ NMR spectra show that the two tBu groups must have a cis relationship. The pseudoasymmetric center at $\mathrm{C} 3$ almost certainly has the methyl group trans to the two tBu groups $\left({ }^{3} \mathrm{~J}_{\mathrm{HH}}=8.4\right) .{ }^{1} \mathrm{H}$ NMR $\left(\mathrm{CDCl}_{3}\right) 0.96(\mathrm{~s}, 18 \mathrm{H}), 1.37(\mathrm{~d}, \mathrm{~J}=6.4,3 \mathrm{H}), 2.18(\mathrm{~m}, 1 \mathrm{H})$, $2.4(\mathrm{~d}, \mathrm{~J}=8.4,2 \mathrm{H}),{ }^{13} \mathrm{C}$ NMR $23.58\left(\mathrm{CH}_{3}\right), 25.41(\mathrm{CH}), 27.8(\mathrm{tBu}), 32.6(\mathrm{C}), 73.49(\mathrm{CH})$, 212.15 (CO); high resolution mass for $\mathrm{C}_{13} \mathrm{H}_{24} \mathrm{O}$, found 196.18383, calcd 196.18272 MS $(\mathrm{m} / \mathrm{z}) 196\left(\mathrm{M}^{+*}, 3\right), 98(20), 83(100), 69(25), 55(5), 41(4)$.

Coordinates for B3LYP/6-31G* Intrinsic Reaction Coordinate Calculation (IRC) where the carbonyl out-of-plane distortion is at a maximum.

\begin{tabular}{lccc}
\multicolumn{1}{c}{$\mathrm{X}$} & $\mathrm{Y}$ & $\mathrm{Z}$ \\
6 & -0.328223 & -0.000006 & -0.189299 \\
6 & 0.798186 & 0.874857 & 0.051470 \\
1 & 1.494239 & 1.174705 & -0.724099 \\
1 & 0.763089 & 1.505594 & 0.938723 \\
8 & -1.515476 & -0.000015 & 0.011086 \\
6 & 0.798201 & -0.874832 & 0.051529 \\
1 & 0.763058 & -1.505512 & 0.938707 \\
1 & 1.494440 & -1.174782 & -0.72421 \\
References & & & \\
1. Mantell, D.R.; Gladfelter, W.L. J.Organomet. Chem. 1998, 347, 333. \\
2. Conia, J-M.; Salaün, J. Bull. Soc. Chem. Fr. 1965, 2747,2751. \\
3. Leusen, D.; Van, A.M. Synthesis 1980 4 325. \\
4. Gaussian 03, Revision A.1, Frisch, M. J.; Trucks, G. W.; Schlegel, H. B.; Scuseria, G. E.; \\
Robb, M. A.; Cheeseman, J. R.; Montgomery, Jr., J. A.; Vreven, T.; Kudin, K. N.; Burant, J. C.; \\
Millam, J. M.; Iyengar, S. S.; Tomasi, J.; Barone, V.; Mennucci, B.; Cossi, M.; Scalmani, G.;
\end{tabular}


Rega, N.; Petersson, G. A.; Nakatsuji, H.; Hada, M.; Ehara, M.; Toyota, K.; Fukuda, R.; Hasegawa, J.; Ishida, M.; Nakajima, T.; Honda, Y.; Kitao, O.; Nakai, H.; Klene, M.; Li, X.;

Knox, J. E.; Hratchian, H. P.; Cross, J. B.; Adamo, C.; Jaramillo, J.; Gomperts, R.; Stratmann, R. E.; Yazyev, O.; Austin, A. J.; Cammi, R.; Pomelli, C.; Ochterski, J. W.; Ayala, P. Y.; Morokuma, K.; Voth, G. A.; Salvador, P.; Dannenberg, J. J.; Zakrzewski, V. G.; Dapprich, S.; Daniels, A. D.; Strain, M. C.; Farkas, O.; Malick, D. K.; Rabuck, A. D.; Raghavachari, K.; Foresman, J. B.; Ortiz, J. V.; Cui, Q.; Baboul, A. G.; Clifford, S.; Cioslowski, J.; Stefanov, B. B.; Liu, G.; Liashenko, A.; Piskorz, P.; Komaromi, I.; Martin, R. L.; Fox, D. J.; Keith, T.; Al-Laham, M. A.; Peng, C. Y.; Nanayakkara, A.; Challacombe, M.; Gill, P. M. W.; Johnson, B.; Chen, W.; Wong, M. W.; Gonzalez, C.; and Pople, J. A.; Gaussian, Inc., Pittsburgh PA, 2003 


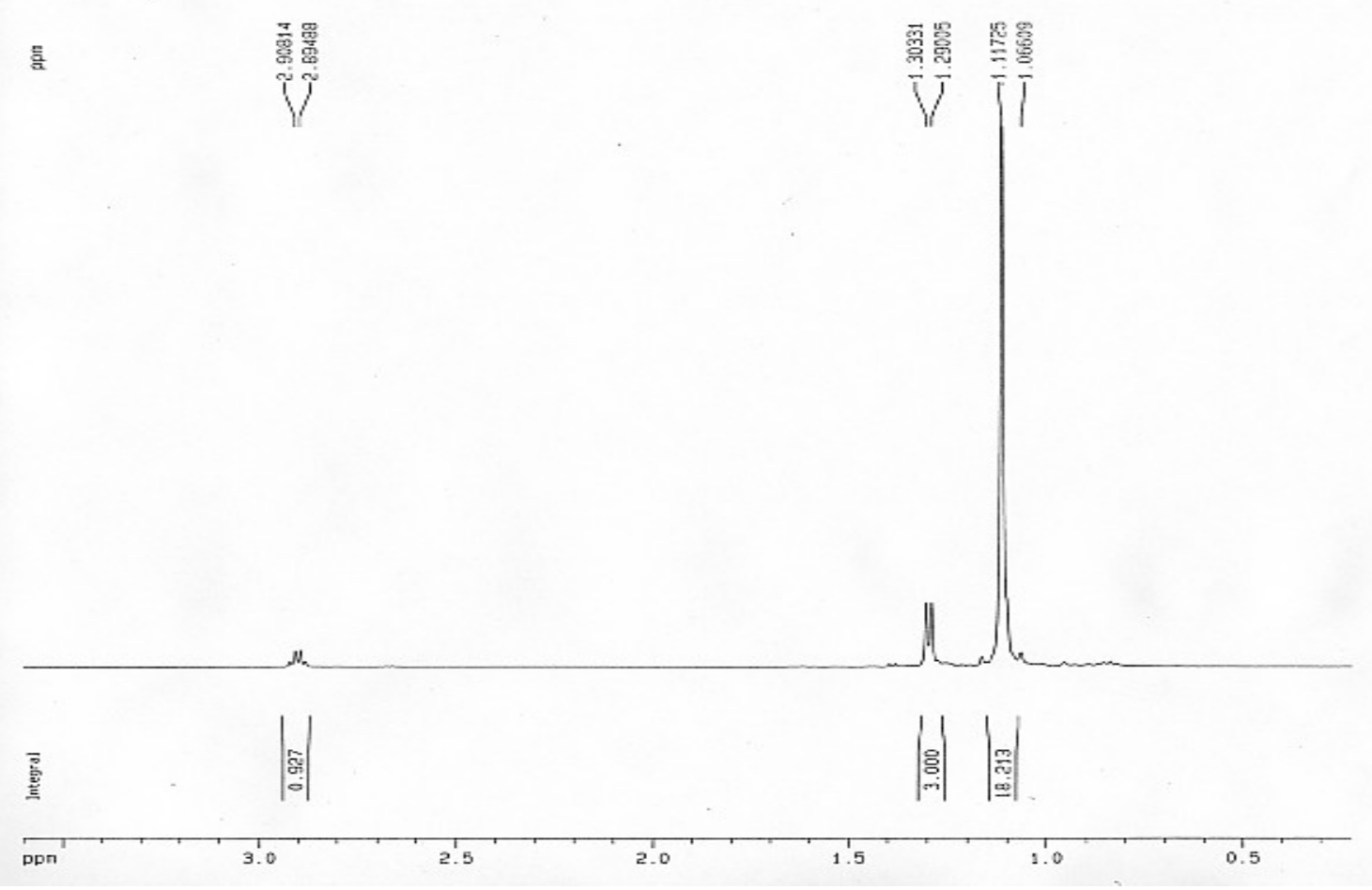

4- ${ }^{1} \mathrm{H}$ NMR

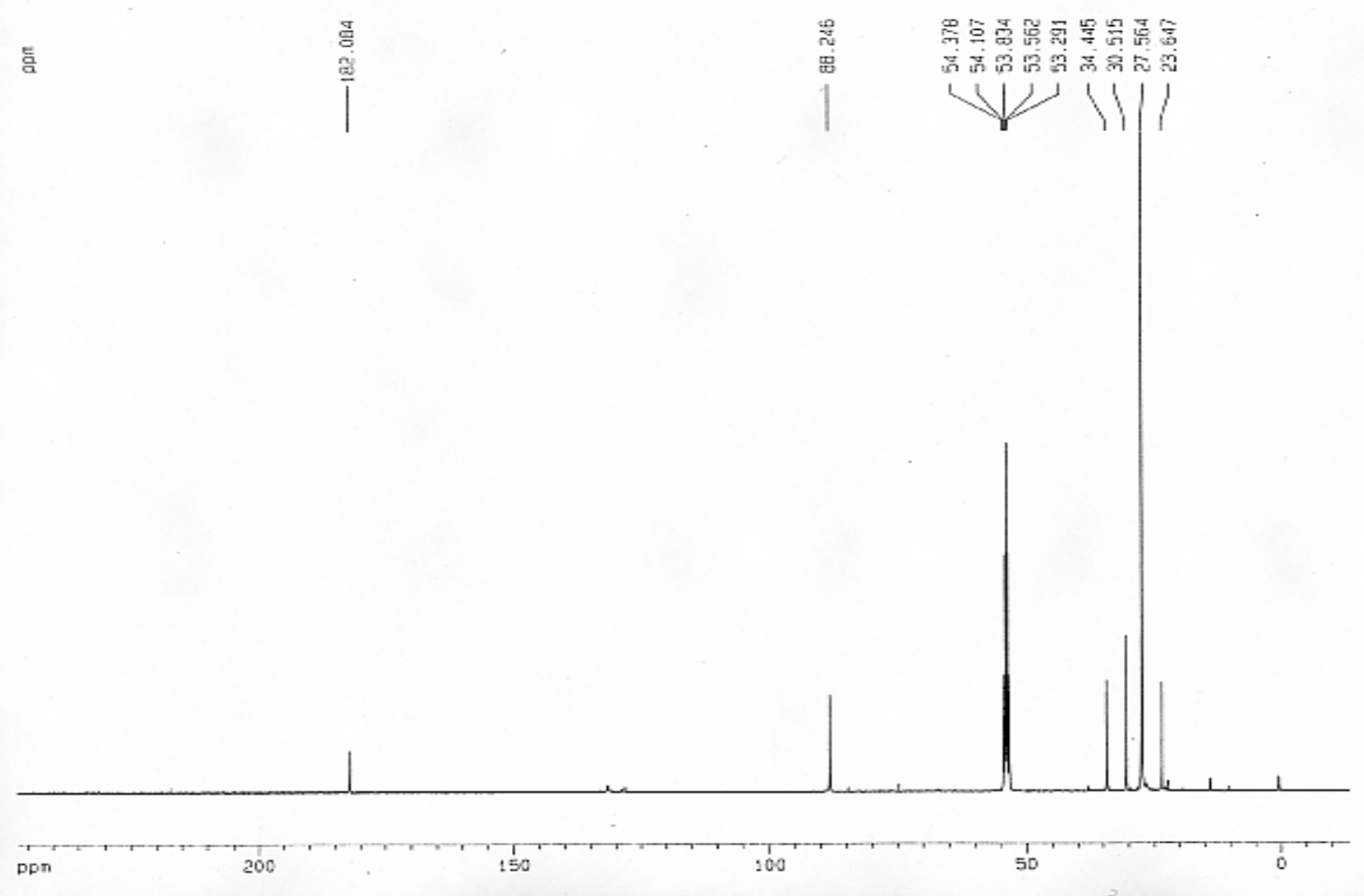

4- ${ }^{13} \mathrm{C}$ NMR 


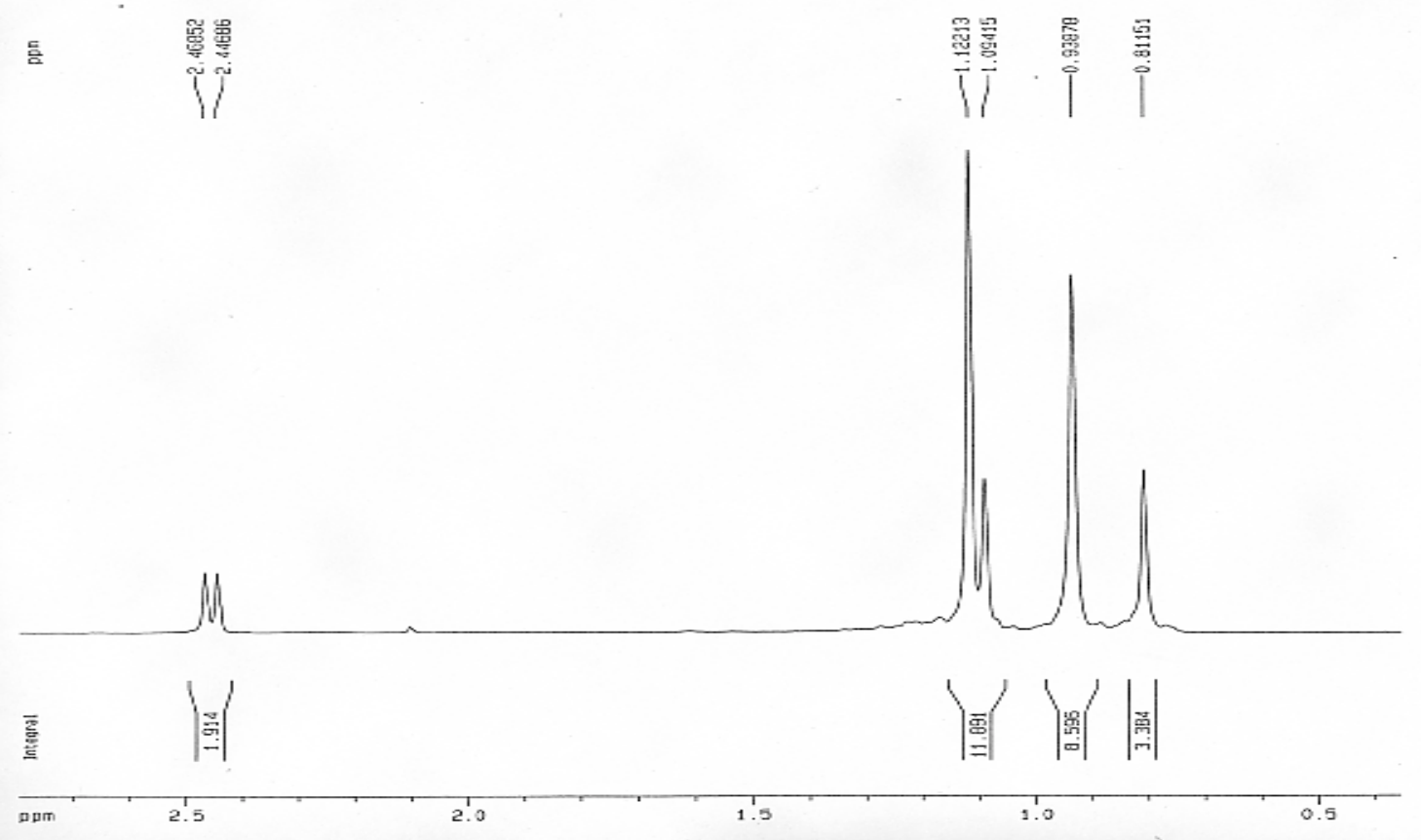

\section{5- ${ }^{1} \mathrm{H}$ NMR}
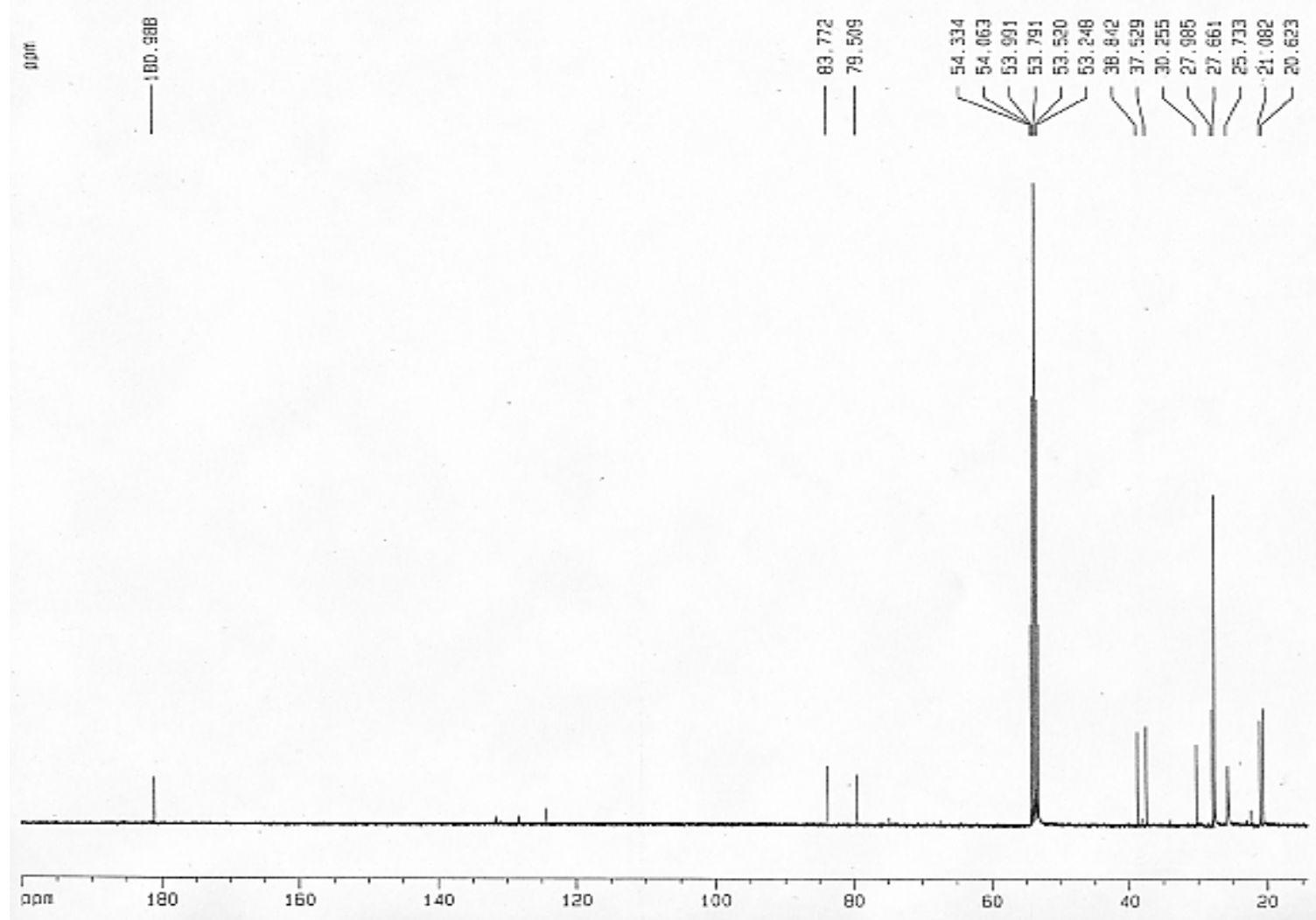

5- ${ }^{13} \mathrm{C}$ NMR 


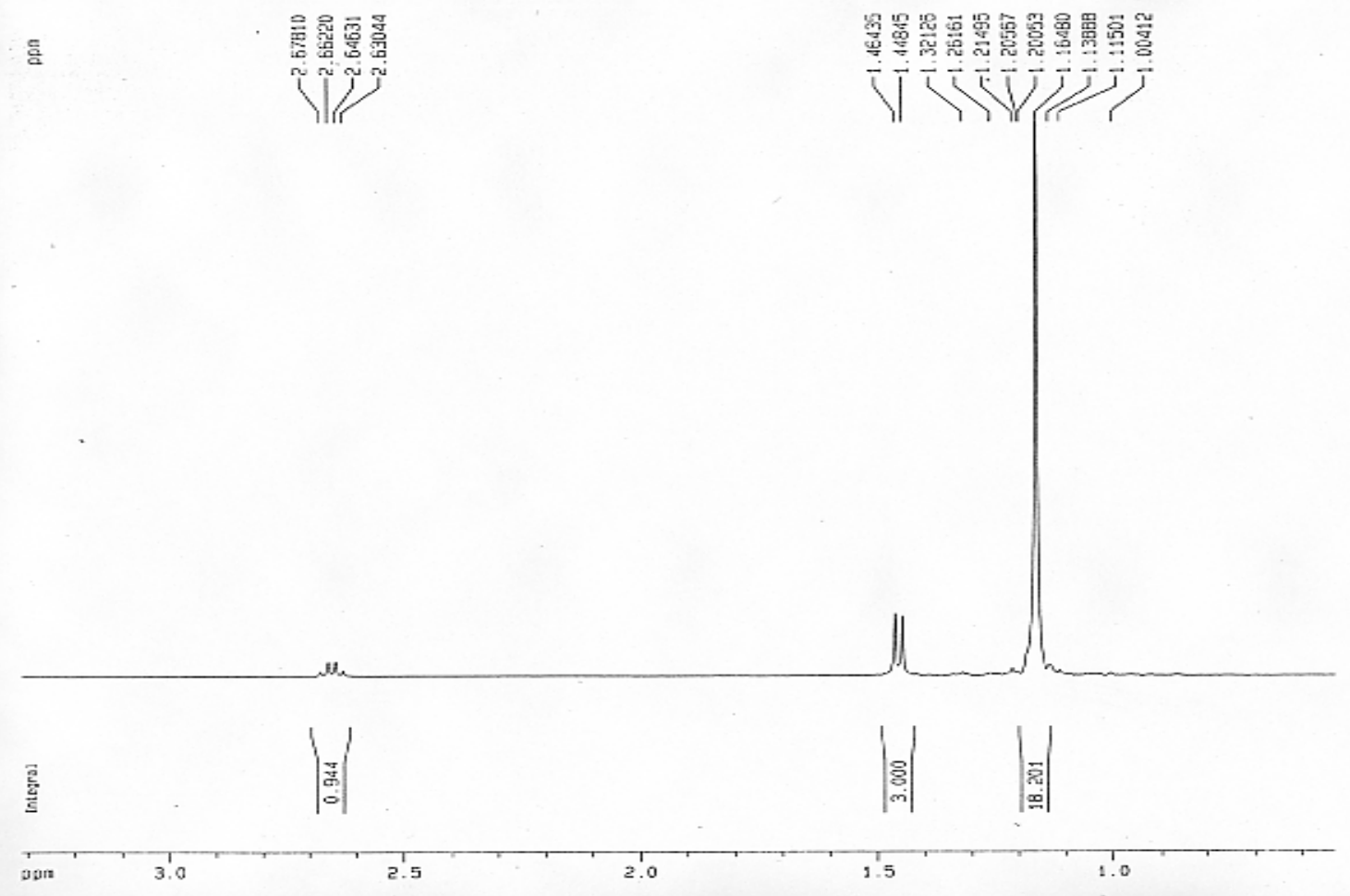

6- ${ }^{1} \mathrm{H}$ NMR

ह

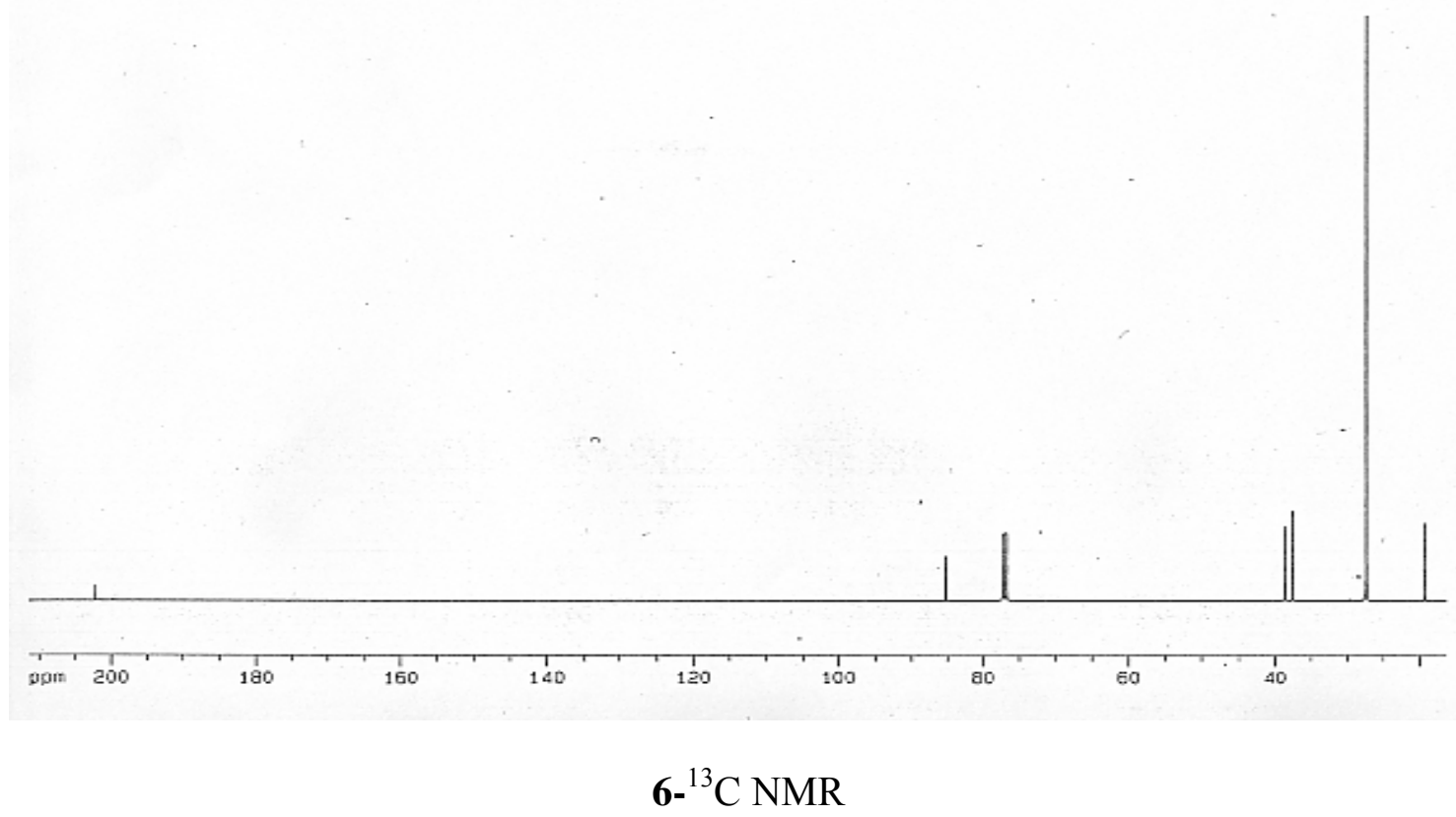




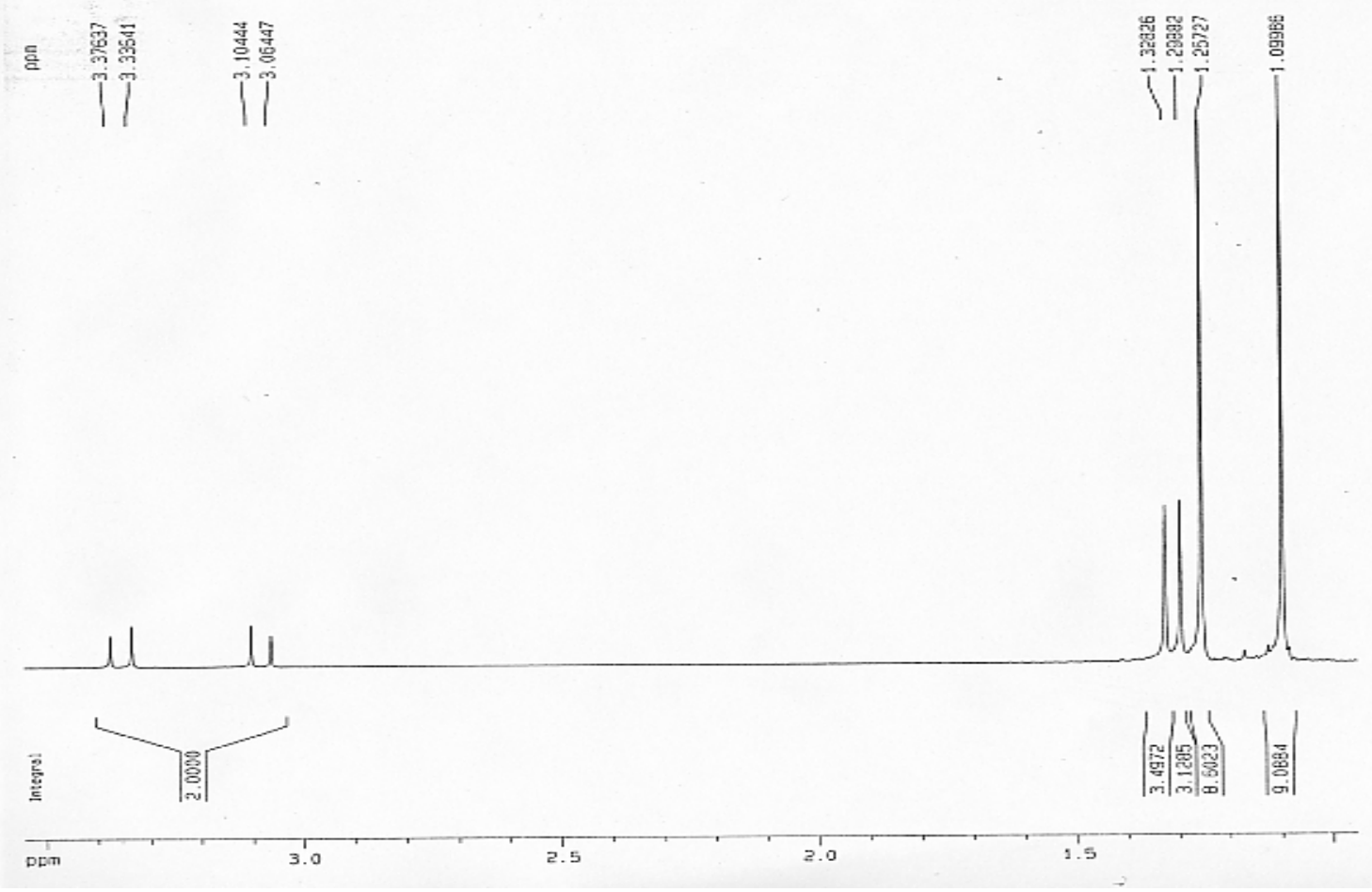

7- ${ }^{1} \mathrm{H}$ NMR

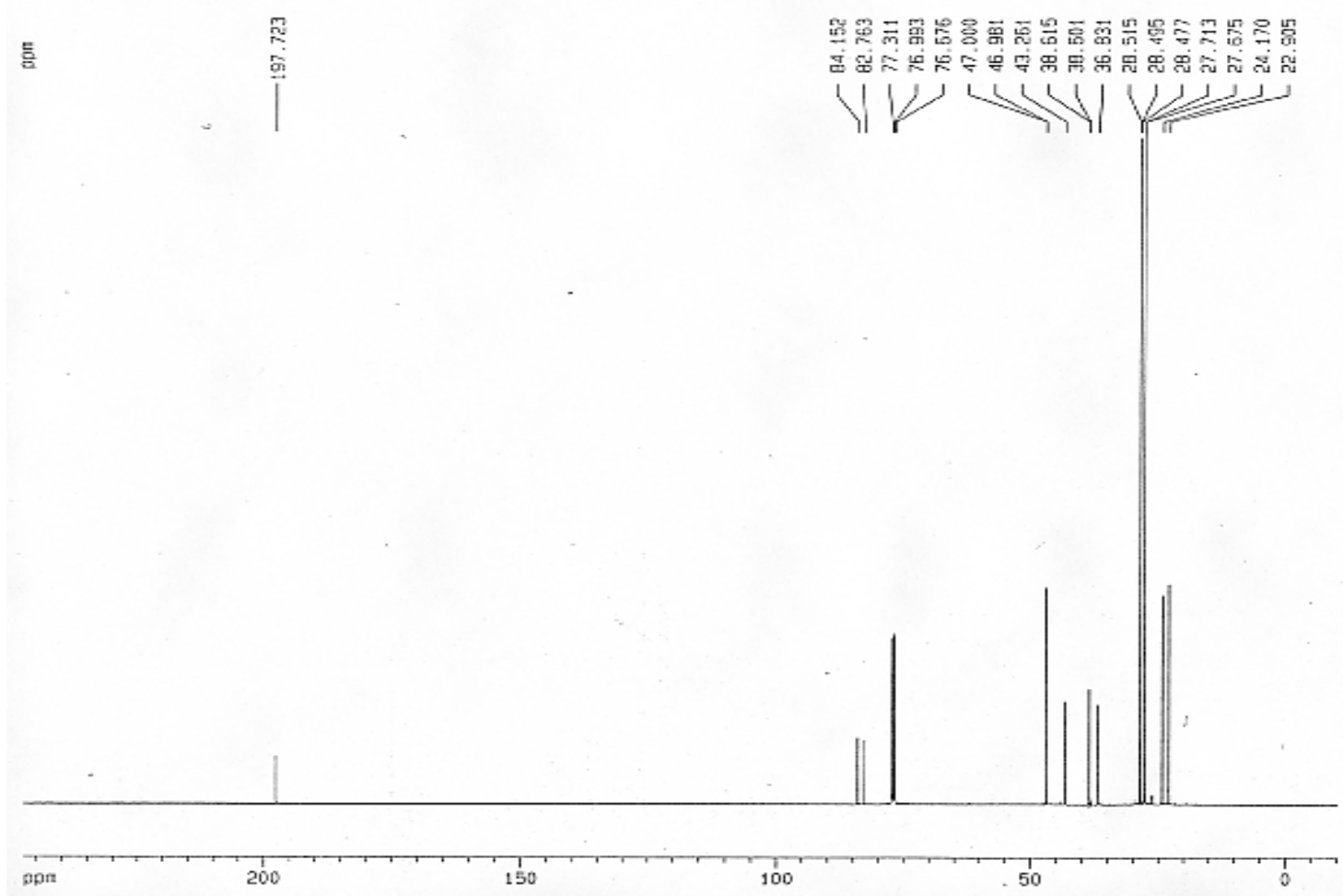

7- ${ }^{13} \mathrm{C}$ NMR 


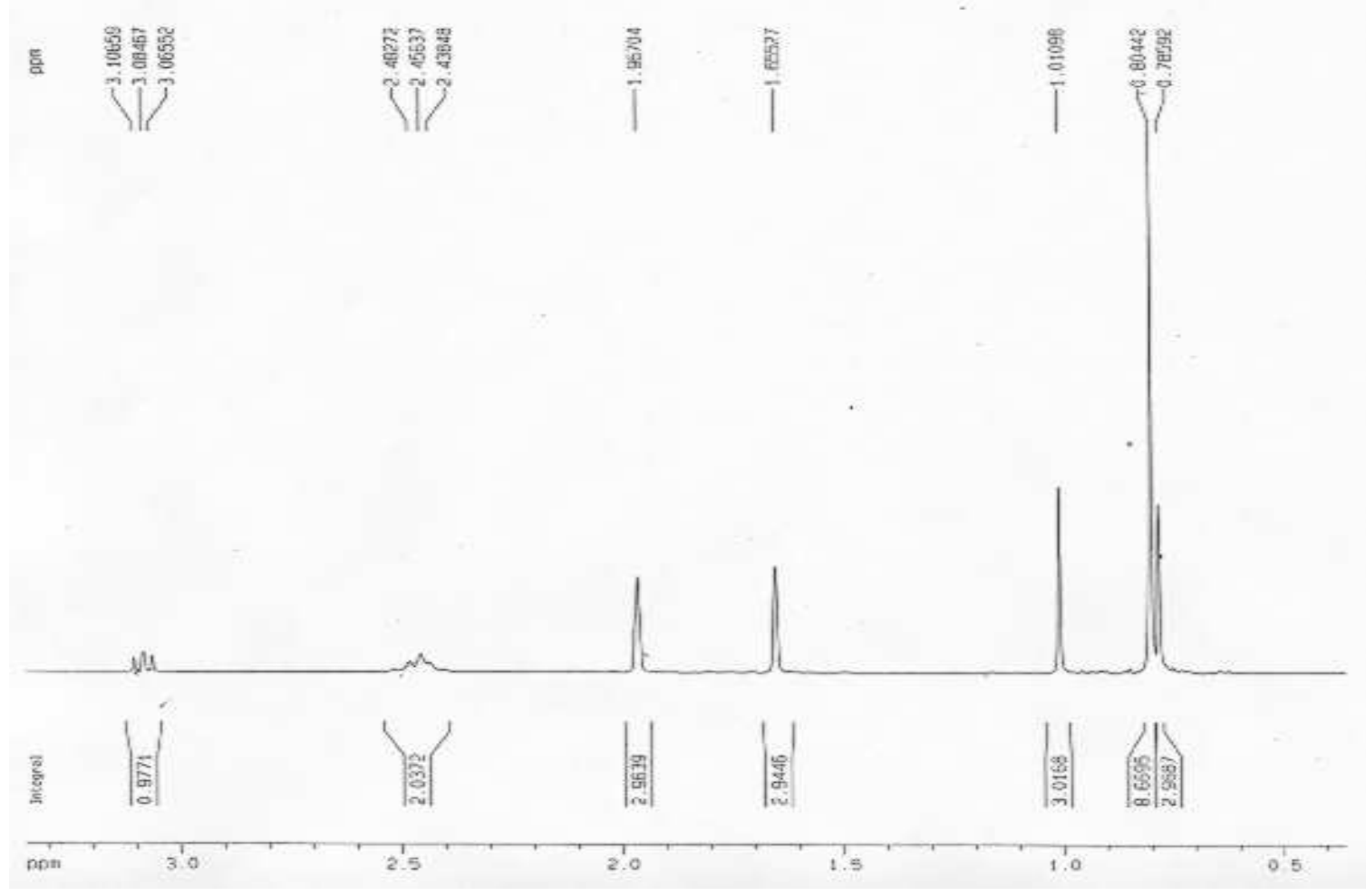

\section{8- ${ }^{1} \mathrm{H}$ NMR}

ร
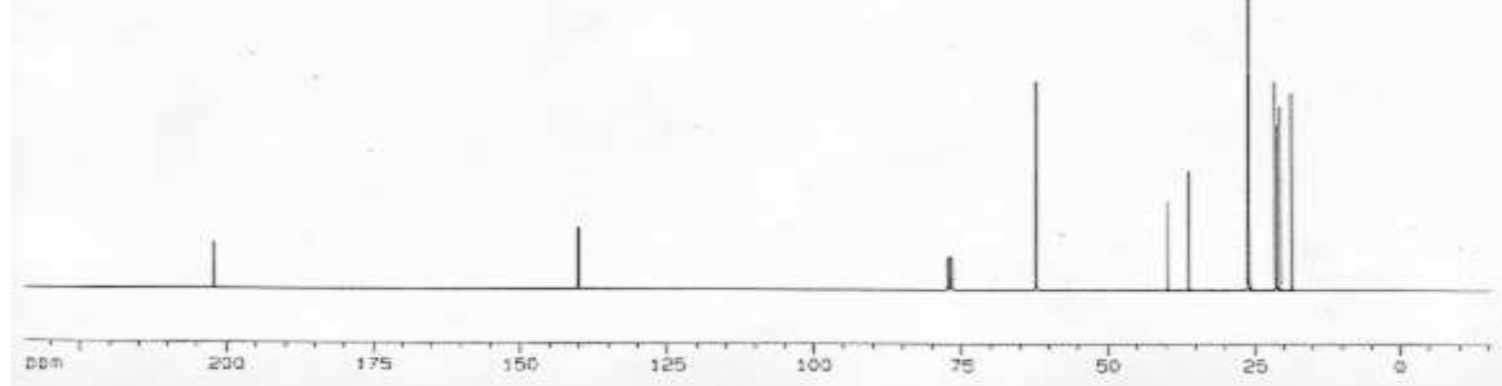

8- ${ }^{13} \mathrm{C}$ NMR

S-10 


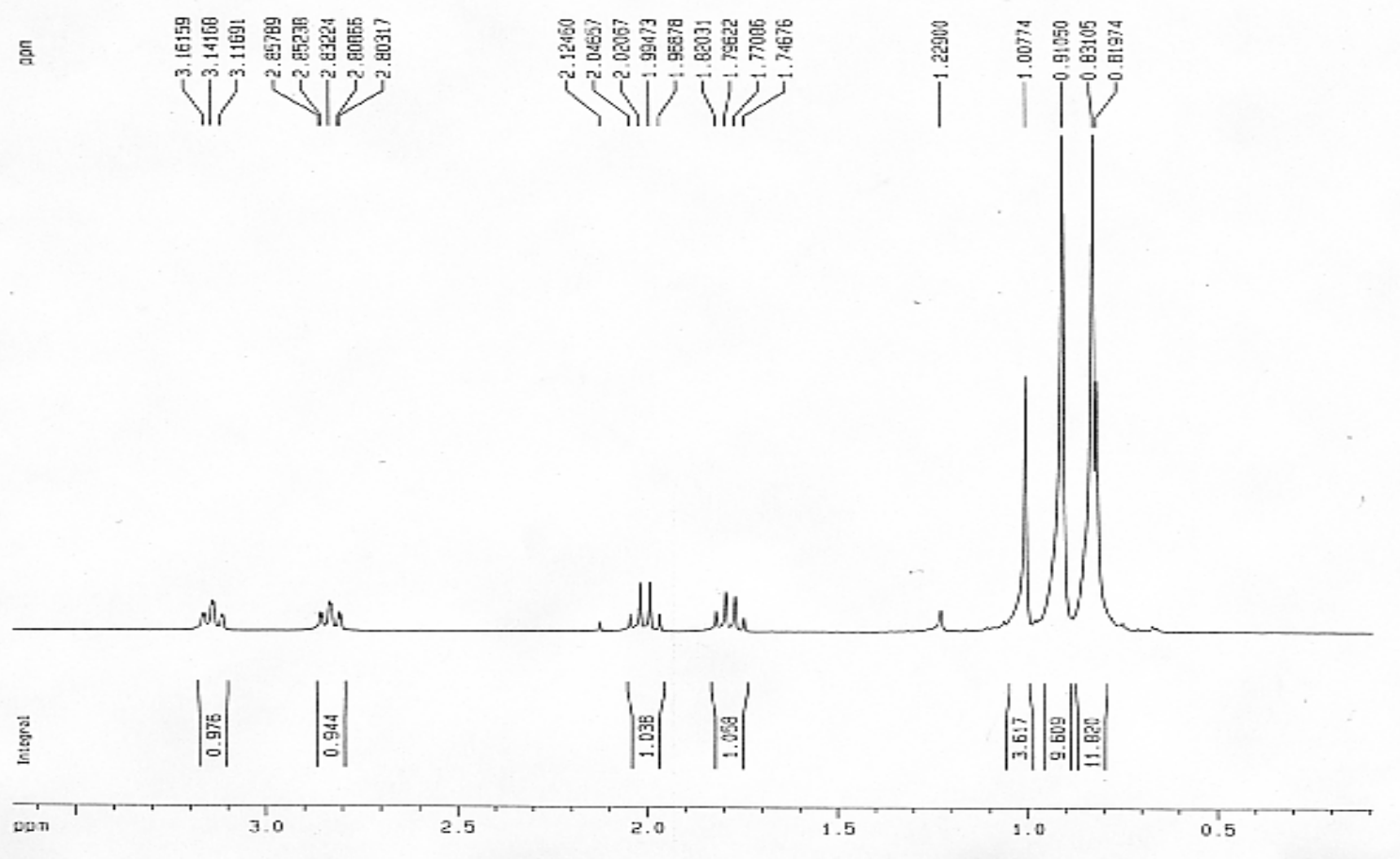

9- ${ }^{1} \mathrm{H}$ NMR

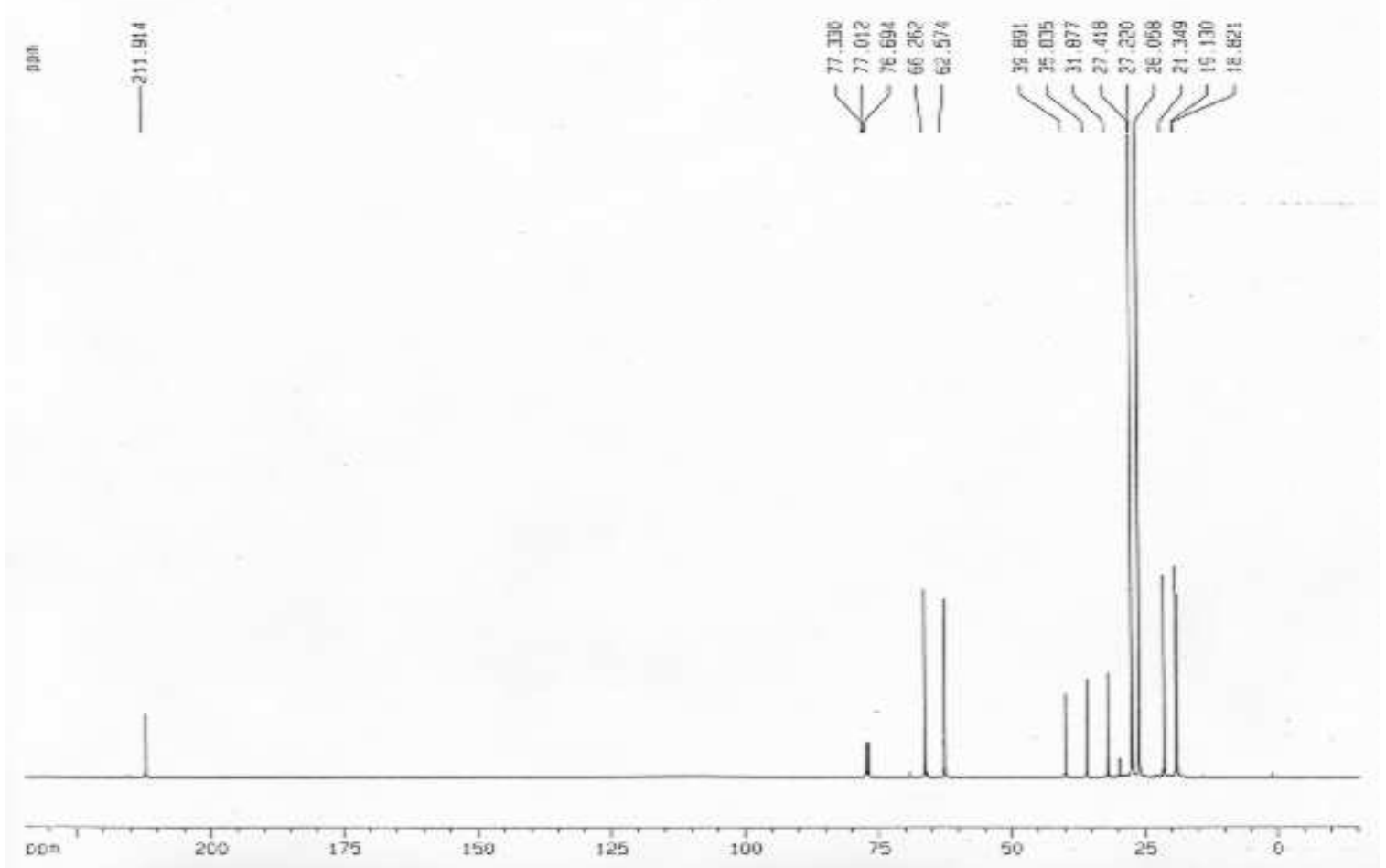

9. ${ }^{13} \mathrm{C}$ NMR 


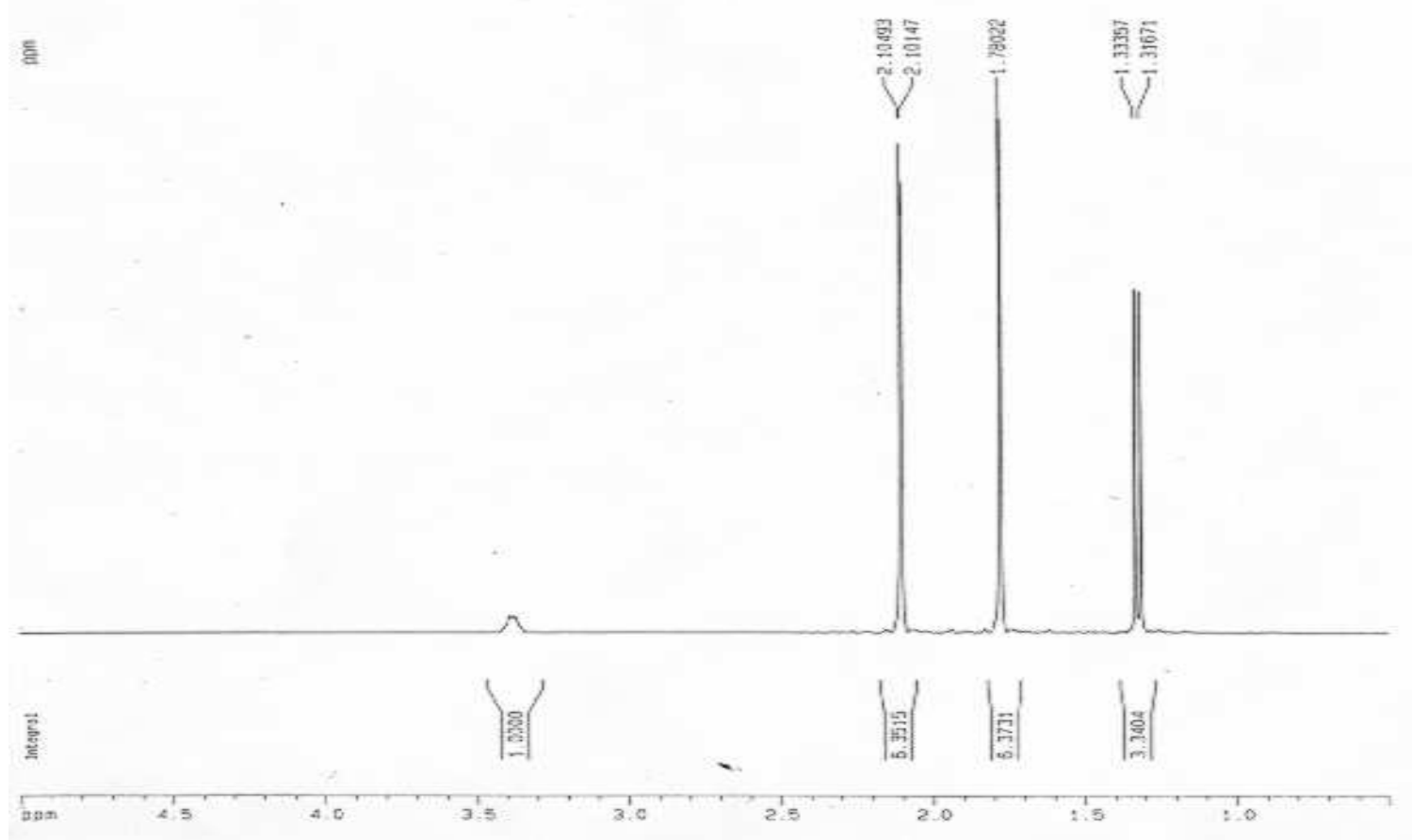

\section{0- ${ }^{1} \mathrm{H}$ NMR}

|

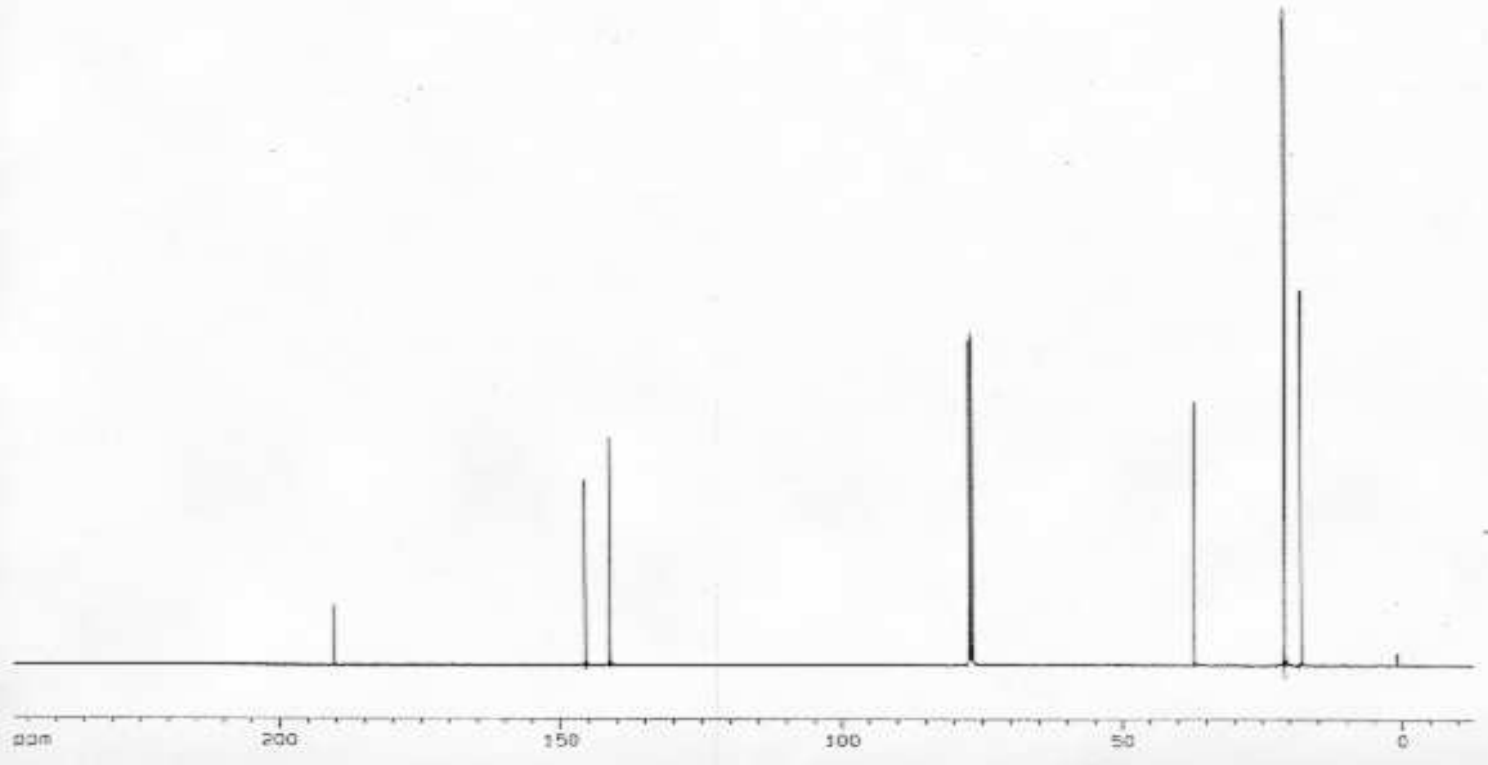

10- ${ }^{13} \mathrm{C}$ NMR

S-12 


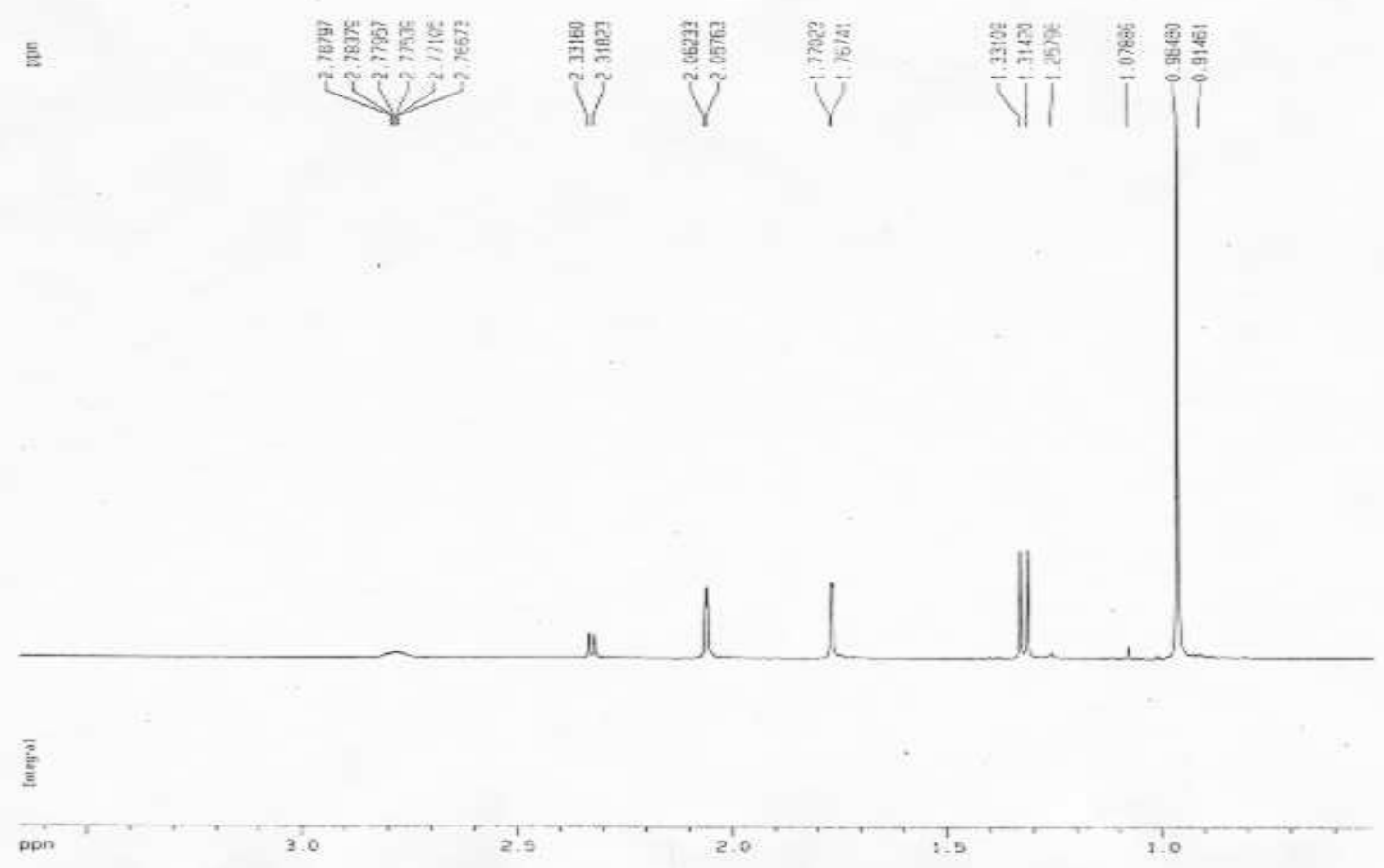

11- ${ }^{1} \mathrm{H}$ NMR

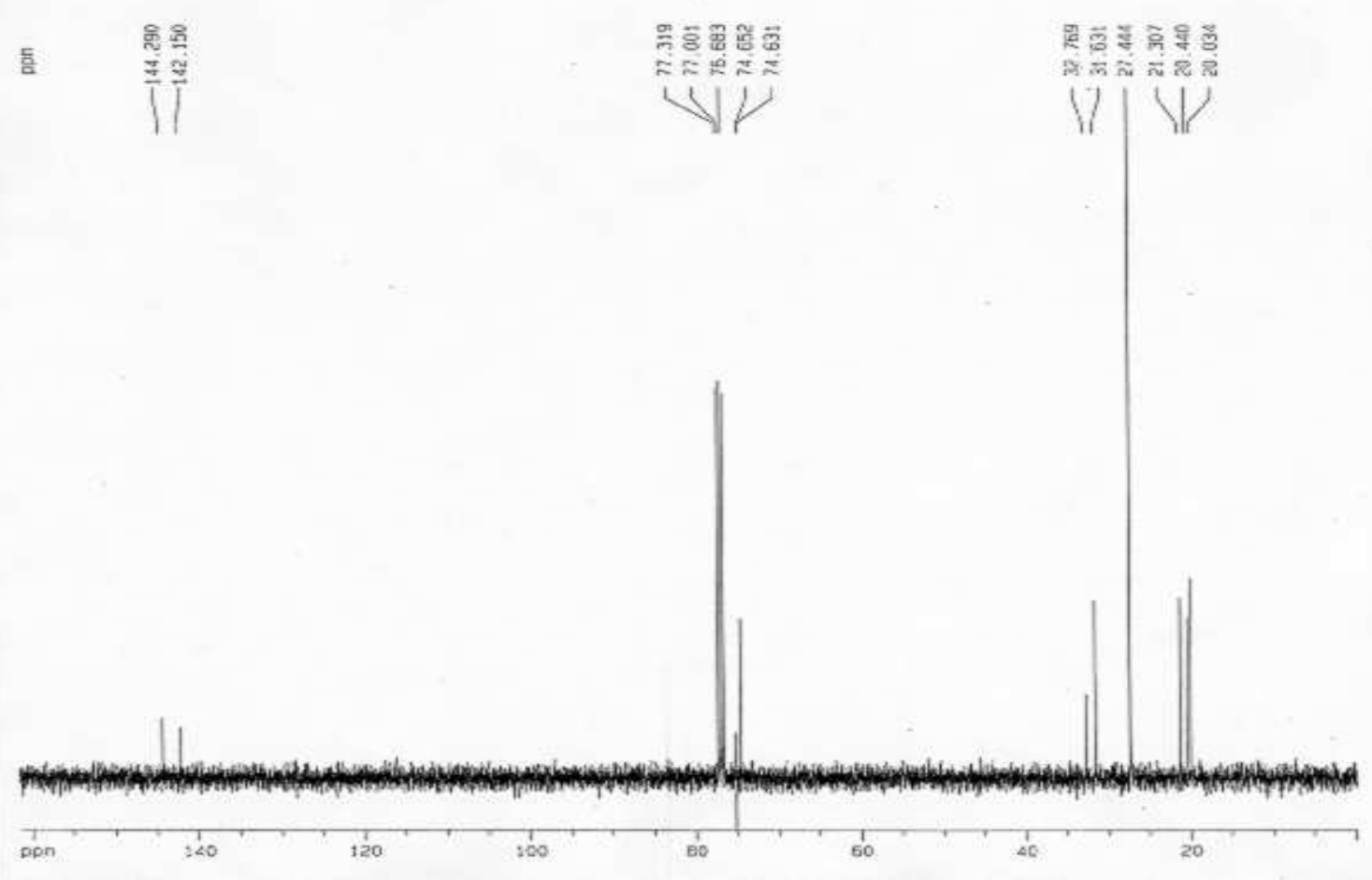

11- ${ }^{13} \mathrm{C}$ NMR

S-13 

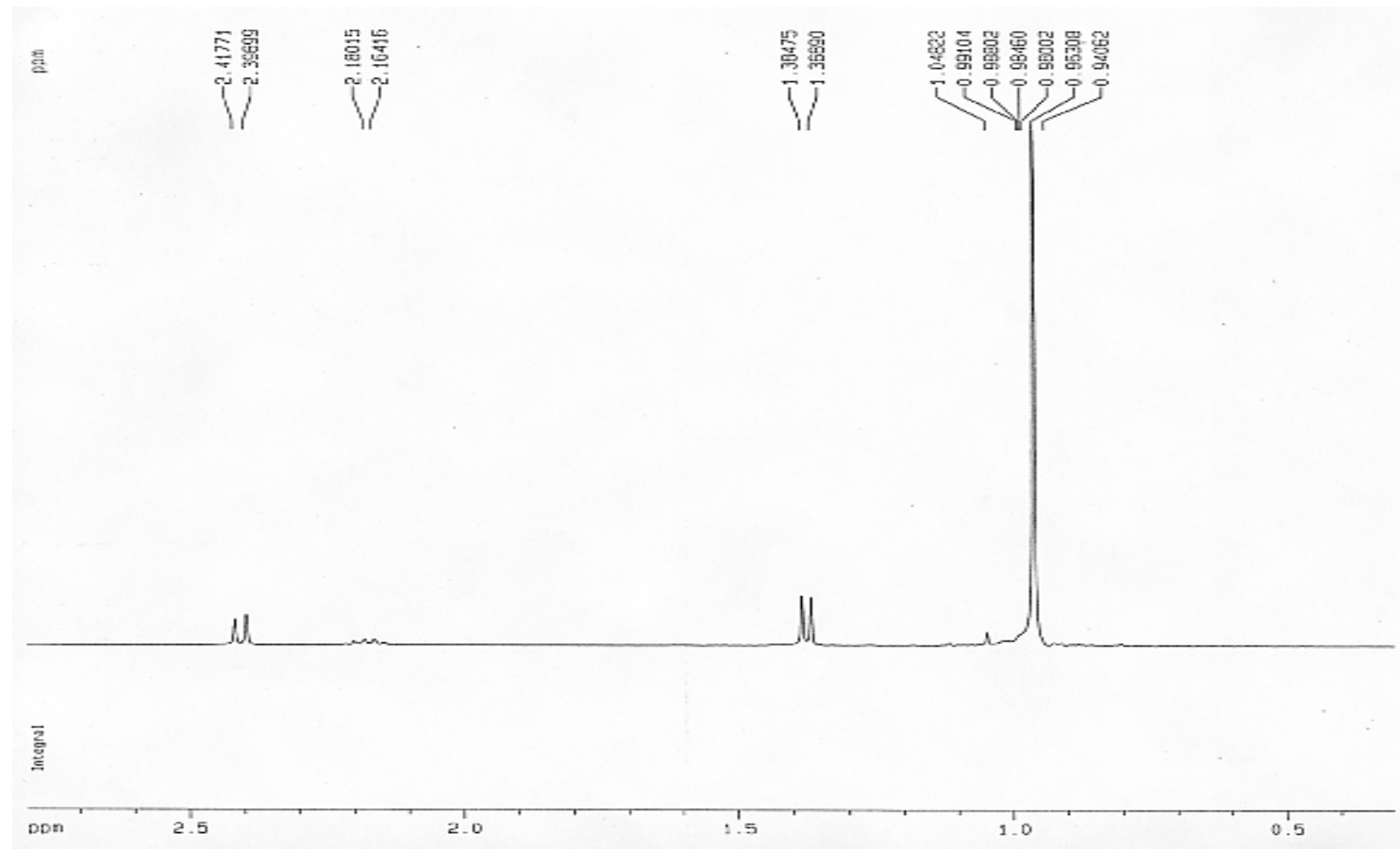

12- ${ }^{1} \mathrm{H}$ NMR
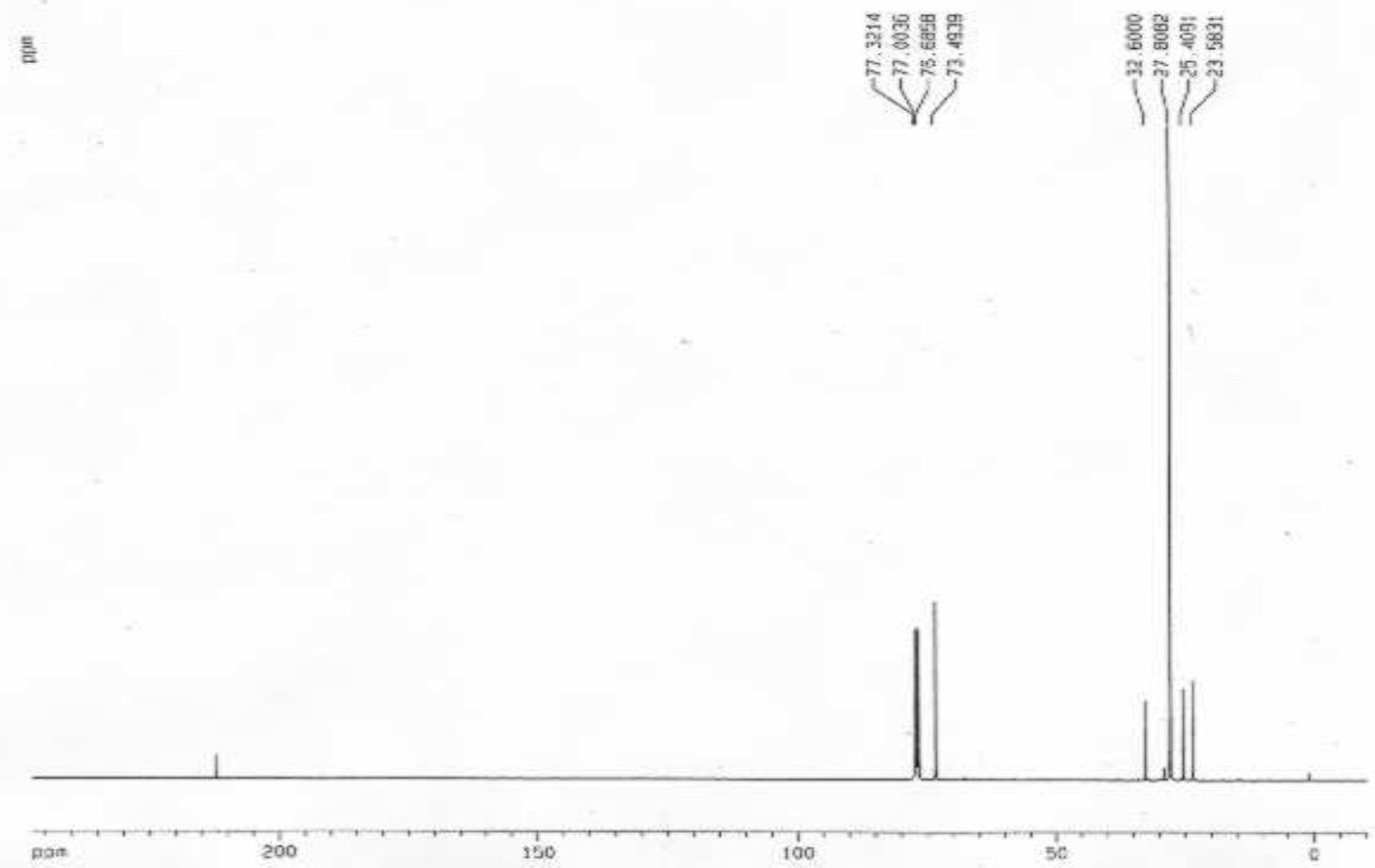

12- ${ }^{13} \mathrm{C}$ NMR 